\title{
Etude in situ par EXAFS de la réductibilité d'un catalyseur Fischer-Tropsch à base de cobalt supporté sur silice
}

\author{
A. Bensaddik, B. Ernst, F. Garin, G. Maire et A. Kiennemann \\ L.E.R.C.S.I., URA 1498, 1 rue Blaise Pascal, 67008 Strasbourg cedex, France
}

\begin{abstract}
Résumé : La réduction sous flux d'hydrogène d'un catalyseur, $25 \% \mathrm{Co} / \mathrm{SiO}_{2}$, pour la synthèse d'hydrocarbures a été suivie par spectroscopie d'absorption des rayons X (EXAFS) in situ. Les analyses numériques EXAFS ont permis d'identifier la phase spinelle $\mathrm{Co}_{3} \mathrm{O}_{4}$ du catalyseur avant traitement. La réduction de $\mathrm{Co}_{3} \mathrm{O}_{4}$ en deux étapes, $\mathrm{CoO}$ puis $\mathrm{Co}^{\circ}$, observée en EXAFS, confirme ainsi les études menées en XPS (surface) et en TPR (bulk). Le nombre de coordination du cobalt a été déterminé à chaque étape de réduction.
\end{abstract}

\begin{abstract}
The reduction under hydrogen flow of a $25 \% \mathrm{Co} / \mathrm{SiO}_{2}$ catalyst for the hydrocarbons synthesis have been followed in situ by Extended X-Ray Adsorption Fine Structure Spectroscopy (EXAFS). The analysis of the EXAFS spectra enables the identification of a $\mathrm{Co}_{3} \mathrm{O}_{4}$ spinel phase before treatment. The two step reduction of $\mathrm{Co}_{3} \mathrm{O}_{4}$ to $\mathrm{CoO}$ then $\mathrm{Co}^{\circ}$, as observed by EXAFS, is consistent with the studies in XPS (surface) and TPR (bulk). The coordination number of cobalt has been determined at each reduction step.
\end{abstract}

\section{INTRODUCTION}

La technique EXAFS, appliquée aux études de caractérisation de catalyseurs, offre l'avantage d'effectuer des mesures spectroscopiques dans des conditions de réaction réalistes. Elle permet de suivre l'évolution structurale du système à haute température et sous gaz réactionnel $[1,2]$.

Le domaine de la synthèse Fischer-Tropsch présente, en catalyse hétérogène, un nouvel intérêt avec le développement du procédé de conversion du méthane en gaz de synthèse (CO $\left.+\mathrm{H}_{2}\right)[3,4]$ et la possibilité d'obtenir des carburants diesel "propres". A partir de ces gaz, la synthèse conduit, par rupture de la liaison $\mathrm{C}-\mathrm{O}$, hydrogénation en espèces $\mathrm{CH}_{\mathrm{x}}$ et polymérisation, à la formation d'hydrocarbures linéaires de longueur de chaîne variable. Les catalyseurs mentionnés dans la littérature pour ce type de réaction sont, généralement, à base de cobalt déposé sur un support $\left(\mathrm{SiO}_{2}, \mathrm{Al}_{2} \mathrm{O}_{3}\right)$ jouant le rôle de dispersant [5]. L'espèce active lors de la réaction, est la forme métallique $\mathrm{Co}^{\circ}$. L'étape préalable à la réaction de synthèse est la réduction du catalyseur, qui après préparation et calcination sous air, se trouve sous sa forme oxydée.

L'objet de cette étude est de suivre par EXAFS in-situ, sous flux d'hydrogène, les différentes étapes de réduction dans le bulk d'un catalyseur 25\% (massique) Co supporté sur silice, performant en synthèse Fischer-Tropsch.

\section{PARTIE EXPERIMENTALE}

\section{1. Préparation du système catalytique}

Les précurseurs de cobalt $\left(\mathrm{Co}\left(\mathrm{NO}_{3}\right)_{2}, 6 \mathrm{H}_{2} \mathrm{O}\right)$ et de silicium $\left(\mathrm{Si}(\mathrm{EtO})_{4}\right)$ sont précipités par l'acide oxalique en solution alcoolique. Une évaporation, menée jusqu'au temps de 
mûrissement, correspondant à l'hydrolyse du tetraéthoxysilane par l'eau d'hydratation du nitrate de cobalt et de l'acide oxalique, conduit, après séchage et calcination à $823 \mathrm{~K}$ (pendant 5 heures), à un catalyseur contenant $25 \%$ en masse de cobalt. Il sera noté $25 \% \mathrm{Co} / \mathrm{SiO}_{2}$.

\section{2. Structure et texture du catalyseur}

Après calcination sous air, le catalyseur $25 \% \mathrm{Co} / \mathrm{SiO}_{2}$ se présente sous sa forme oxydée. Sa surface spécifique obtenue par la technique BET [6] est de $393 \mathrm{~m}^{2} / \mathrm{g}$. L'unique structure cristalline détectée en diffraction des rayons X est la spinelle $\mathrm{Co}_{3} \mathrm{O}_{4}$. Le support $\mathrm{SiO}_{2}$, présentant les propriétés d'un gel de silice, est amorphe. La taille moyenne des cristallites d'oxydes de cobalt $\left(\mathrm{Co}_{3} \mathrm{O}_{4}\right)$, calculée d'après l'élargissement des raies de diffraction [7] est de $300 \AA$. Une analyse par microscopie électronique en mode transmission (M.E.T.) a confirmé ce résultat.

\section{3. Dispositif expérimental EXAFS}

Les mesures EXAFS sont réalisées au centre synchrotron de LURE à Orsay, en utilisant le rayonnement électromagnétique issu de l'anneau de stockage DCI (Dispositif de Collision dans l'Igloo). Cet anneau est caractérisé par une énergie de $1.85 \mathrm{GeV}$ et un courant machine de l'ordre de $300 \mathrm{~mA}$. Les spectres EXAFS sont enregistrés au seuil $\mathrm{K}$ du cobalt $(7708,9 \mathrm{eV})$ et le faisceau "blanc" polychromatique est monochromatisé par une double réflexion de Bragg sur des cristaux de silicium $\mathrm{Si}(111)$. Les mesures d'absorption de rayons $\mathrm{X}$ sont réalisées par transmission. On a utilisé comme système de détection deux chambres à ionisation pour mesurer l'intensité du faisceau avant et après traversée de l'échantillon par les photons $\mathrm{X}$. Le catalyseur $25 \% \mathrm{Co} / \mathrm{SiO}_{2}$ est sous forme de poudre finement broyée, la taille des grains est comprise entre 100 et $200 \mu \mathrm{m}$, afin de permettre une meilleure circulation des gaz. Il est placé dans un réacteur qui nous permet de travailler dans un domaine de température relativement large $(290 \mathrm{~K}<\mathrm{T}<900 \mathrm{~K})$, avec la possibilité d'envoyer sur l'échantillon des quantités définies de différents gaz : air, mélange $\left(\mathrm{CO}+\mathrm{H}_{2}\right)$, gaz neutre $\left(\mathrm{N}_{2}, \mathrm{He}, \mathrm{Ar}, \ldots.\right)$, hydrogène $\left(\mathrm{H}_{2}\right)$. Les deux parois du réacteur, à la hauteur du porte-échantillon, sont munies de fenêtres de kapton de $50 \mu \mathrm{m}$ d'épaisseur pour permettre le passage des rayons $\mathrm{X}$ à travers l'échantillon.

Le traitement se déroule in situ, selon un protocole expérimental qui comprend trois étapes : une première réduction de trente minutes sous hydrogène à $473 \mathrm{~K}$, une seconde réduction de trente minutes sous hydrogène à $553 \mathrm{~K}$ et finalement une dernière réduction sous hydrogène à 673K pendant une heure et trente minutes. Ce traitement est destiné à suivre la réduction du catalyseur cobalt sur silice en fonction de la température.

\section{CONDITIONS D'ANALYSE EXAFS}

Pour chaque étape de réduction, les spectres d'absorption au seuil $\mathrm{K}$ du cobalt sont enregistrés après retour à température ambiante pour optimiser le rapport signal/bruit et simplifier l'analyse des données, l'échantillon restant sous balayage du gaz réactant.

Dans ce travail, la feuille de cobalt métallique, la spinelle $\mathrm{Co}_{3} \mathrm{O}_{4}$ et $\mathrm{CoO}$ sont utilisés comme références pour la simulation des liaisons Co-Co et Co-O. La transformée de Fourier de l'oscillation EXAFS est obtenue en utilisant une fenêtre de Hanning de $12 \AA$ \& 1 . Par une transformée de Fourier inverse, avec un filtre entre 1,61-3,21 $\AA$, on obtient la modulation EXAFS filtrée relative à la couche que l'on veut analyser. La détermination des paramètres structuraux (le nombre de voisins et la distance interatomique) se fait par l'utilisation des fonctions phase et amplitude des différents composés de références, dont les structures cristallographiques sont connues et très voisines de celles suspectées exister dans l'échantillon lors de l'enregistrement du spectre EXAFS. Tous les affinements sont réalisés dans l'espace des énergies (E) et l'espace des distances $(R)$. [8].

Une description complète de la procédure classique de l'analyse EXAFS est donnée par Teo 


\section{RESULTATS}

\subsection{Catalyseur $25 \% \mathrm{Co} / \mathrm{SiO}_{2}$ avant traitement}

Le spectre d'absorption collecté pour l'échantillon (chargé dans la cellule), avant traitement sous hydrogène, est visualisé sur la figure 1 . Il rend compte de la qualité des données durant l'ensemble de ce travail.

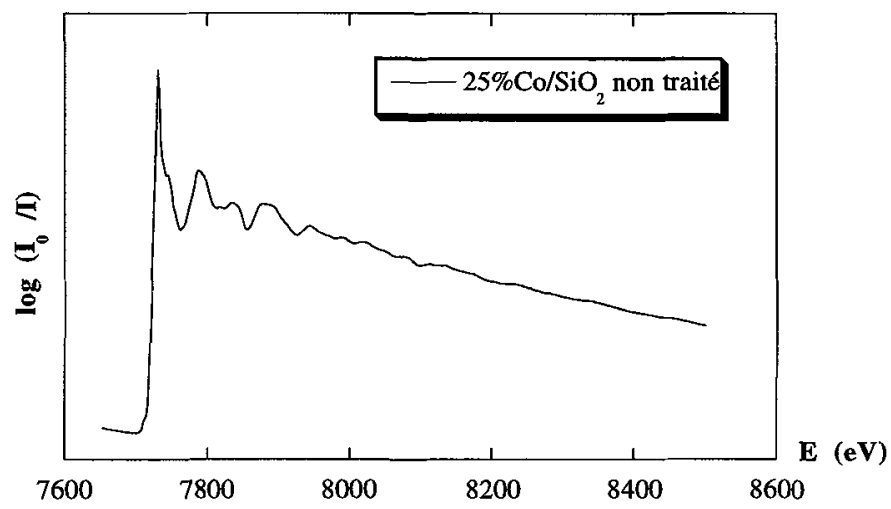

Figure 1: Spectre d'absorption $\mathrm{X}$ (seuil $\mathrm{K}$ du cobalt) du catalyseur $25 \% \mathrm{Co} / \mathrm{SiO}_{2}$ non traité $\mathrm{X}$-ray absorption spectrum (cobalt $\mathrm{K}$ edge) for untreated $25 \% \mathrm{Co} / \mathrm{SiO}_{2}$ catalyst

L'analyse de ce spectre d'absorption EXAFS enregistré au seuil $\mathrm{K}$ du cobalt nous permet de rendre compte de l'état d'oxydation du cobalt. En effet une comparaison du module de la transformée de Fourier de notre catalyseur calciné avec ceux des deux références oxydes de cobalt $\left(\mathrm{Co}_{3} \mathrm{O}_{4}\right.$ et $\left.\mathrm{CoO}\right)$ (figure 2) indique une parfaite similitude avec le module de la transformée de Fourier de la référence $\mathrm{Co}_{3} \mathrm{O}_{4}$. Le cobalt de notre catalyseur, avant tout traitement, est donc totalement sous la forme oxyde (spinelle $\mathrm{Co}_{3} \mathrm{O}_{4}$ ).

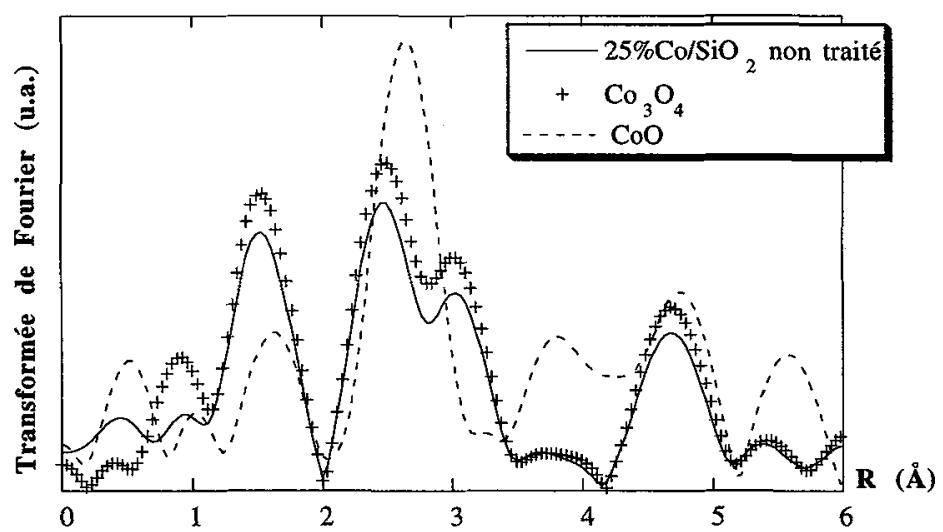

Figure 2: Comparaison entre le module de la transformée de Fourier (seuil $\mathrm{K}$ du cobalt) du catalyseur non traité et ceux des deux composés de références d'oxydes de cobalt $\left(\mathrm{Co}_{3} \mathrm{O}_{4}\right.$ et $\left.\mathrm{CoO}\right)$ 
Comparison between the module of the Fourier transform (cobalt $\mathrm{K}$ edge) of the untreated $25 \% \mathrm{Co} / \mathrm{SiO}_{2}$ catalyst and model compounds $\left(\mathrm{Co}_{3} \mathrm{O}_{4}\right.$ et $\left.\mathrm{CoO}\right)$

\subsection{Catalyseur $25 \% \mathrm{Co} / \mathrm{SiO}_{2}$ réduit sous hydrogène à $473 \mathrm{~K}$}

La première étape de réduction se fait à une température de $473 \mathrm{~K}$ pendant une demi heure. Le module de la transformée de Fourier correspondant, comparé à celui du catalyseur avant traitement, est donné sur la figure 3. La similitude entre ces deux modules montre bien que, après cette réduction à $473 \mathrm{~K}$, le cobalt est toujours sous forme oxyde $\left(\mathrm{Co}_{3} \mathrm{O}_{4}\right)$ et que sa réduction n'est pas encore amorcée.

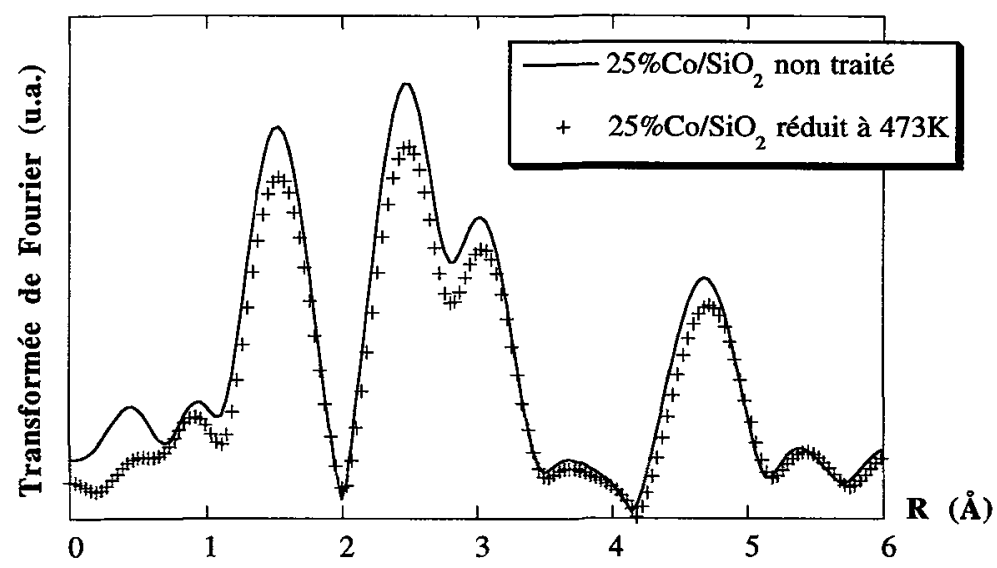

Figure 3: Comparaison entre les deux modules de la transformée de Fourier (seuil $\mathrm{K}$ du cobalt) du catalyseur non traité et après une réduction à $473 \mathrm{~K}$

Comparison between Fourier transforms (cobalt $\mathrm{K}$ edge) for the untreated and reduced at $473 \mathrm{~K}$ $25 \% \mathrm{Co} / \mathrm{SiO}_{2}$ catalyst

\subsection{Catalyseur $25 \% \mathrm{Co} / \mathrm{SiO}_{2}$ réduit sous hydrogène à $553 \mathrm{~K}$}

Après cette deuxième étape, la différence entre le module de la transformée de Fourier correspondant et les modules des étapes précédentes (figure 4), nous permet de rendre compte qualitativement d'un changement dans l'environnement du cobalt. 


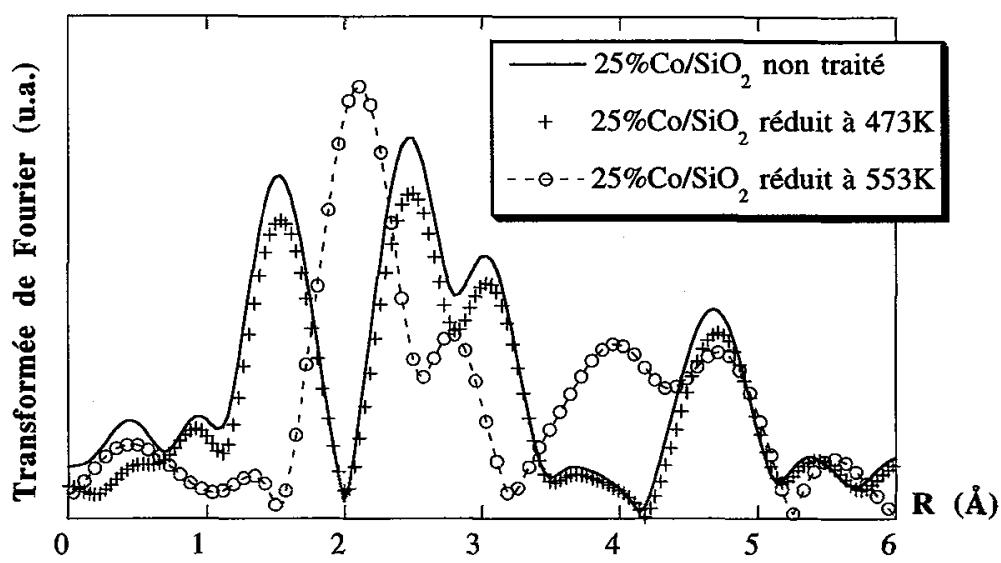

Figure 4: Comparaison entre les modules de la transformée de Fourier (seuil $\mathrm{K}$ du cobalt) du catalyseur $25 \% \mathrm{Co} / \mathrm{SiO}_{2}$ non traité, réduit à $473 \mathrm{~K}$ et réduit à $553 \mathrm{~K}$

Comparison between Fourier transforms (cobalt $\mathrm{K}$ edge) for the untreated, reduced at $473 \mathrm{~K}$, reduced at $553 \mathrm{~K} 25 \% \mathrm{Co} / \mathrm{SiO}_{2}$ catalyst

La confirmation est donnée par l'analyse quantitative EXAFS effectuée en utilisant trois références, la feuille de cobalt métallique, la spinelle $\mathrm{Co}_{3} \mathrm{O}_{4}$ et $\mathrm{CoO}$. Le tableau 1 regroupe les résultats de cette analyse.

Tableau 1: Paramètres structuraux au seuil $\mathrm{K}$ du cobalt du catalyseur $25 \% \mathrm{Co} / \mathrm{SiO}_{2}$ réduit à $553 \mathrm{~K}$ EXAFS parameters (cobalt $\mathrm{K}$ edge) for $25 \% \mathrm{Co} / \mathrm{SiO}_{2}$ catalyst reduced at $553 \mathrm{~K}$

\begin{tabular}{|c|c|c|c|}
\hline $\begin{array}{c}\text { Références } \\
\text { utilisées }\end{array}$ & $\begin{array}{c}\text { Nombre et nature } \\
\text { des voisins }\end{array}$ & $\begin{array}{c}\text { Distances } \\
(\tilde{A})\end{array}$ & $\begin{array}{c}\text { Facteur de } \\
\text { Debye-Waller }\end{array}$ \\
\hline $\mathrm{Co}_{3} \mathrm{O}_{4}$ & $\mathrm{~N}_{\mathrm{Co}-\mathrm{O}}=0,7$ & $\mathbf{R}_{\mathrm{Co}-\mathrm{O}}=1,91$ & $\Delta \sigma=0,03$ \\
\hline $\mathrm{CoO}$ & $\begin{array}{l}\mathrm{N}_{\mathrm{Co}-\mathrm{O}}=3,0 \\
\mathrm{~N}_{\mathrm{Co}-\mathrm{Co}}=3,4\end{array}$ & $\begin{array}{l}\mathrm{R}_{\mathrm{Co}-\mathrm{O}}=2,10 \\
\mathrm{R}_{\mathrm{Co}-\mathrm{Co}}=3,02\end{array}$ & $\begin{array}{l}\Delta \sigma=0,00 \\
\Delta \sigma=0,00\end{array}$ \\
\hline Cobalt métallique & $\mathrm{N}_{\mathrm{Co}-\mathrm{Co}}=4,7$ & $\mathrm{R}_{\mathrm{Co}-\mathrm{Co}}=2,51$ & $\Delta \sigma=0,00$ \\
\hline
\end{tabular}

Les liaisons Co-O détectées proviennent essentiellement de la référence $\mathrm{CoO}$. On montre ainsi que la réduction du cobalt est bien amorcée (le nombre de liaison Co-Co provenant de la référence métallique est non négligable). La spinelle $\left(\mathrm{Co}_{3} \mathrm{O}_{4}\right)$ se réduit en monoxyde de cobalt $\mathrm{CoO}$ avant que ce dernier ne soit réduit en cobalt métallique.

\subsection{Catalyseur $25 \% \mathrm{Co} / \mathrm{SiO}_{2}$ réduit sous hydrogène à $673 \mathrm{~K}$}

Le module de la transformée de Fourier correspondant à cette dernière étape du traitement, présente une parfaite similitude avec celui de la référence cobalt métal (figure 5). 


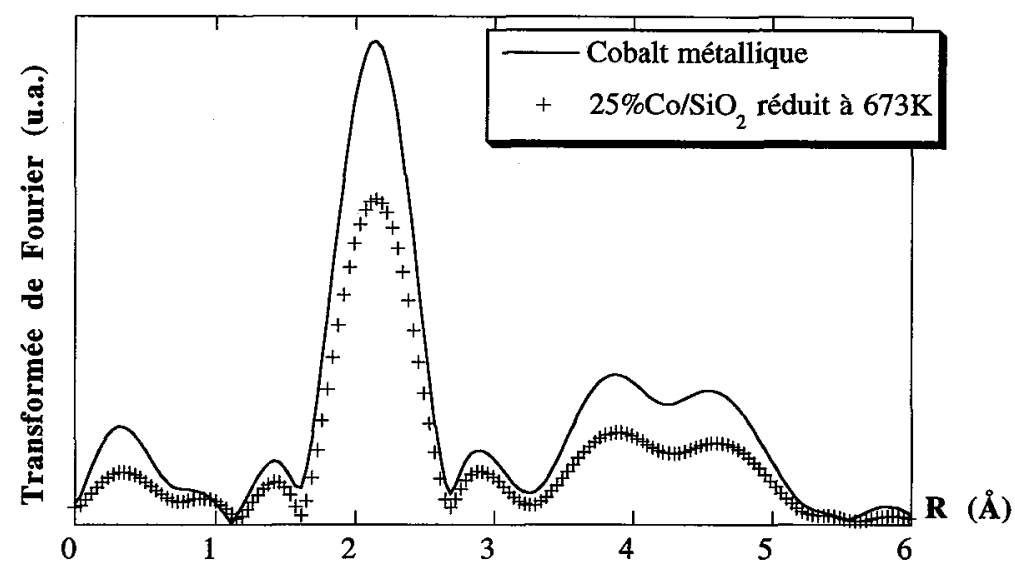

Figure 5: Comparaison entre le module de la transformée de Fourier (seuil K du cobalt) du cobalt métallique et celui du catalyseur réduit à $673 \mathrm{~K}$

Comparison between the module of the Fourier transform (cobalt $\mathrm{K}$ edge) of $25 \% \mathrm{Co} / \mathrm{SiO}_{2}$ reduced at $673 \mathrm{~K}$ catalyst and cobalt metal

Cette similitude est confirmée par la simulation numérique qui donne une première sphère de coordination, pour le cobalt du catalyseur, formée uniquement d'atomes de cobalt $\left(\mathrm{N}_{\mathrm{Co}-\mathrm{Co}}=7,8, \mathrm{R}_{\mathrm{Co}-\mathrm{Co}}=2,51 \AA\right)$. Ce résultat nous montre clairement que le cobalt est réduit dans sa totalité.

\section{DISCUSSION}

Notre étude in situ a permis de mettre en évidence la présence de la phase $\mathrm{Co}_{3} \mathrm{O}_{4}$ avant traitement et l'absence de silicate de cobalt. En effet, le formation de composés de surface avec la silice est limitée [9] contrairement aux catalyseurs cobalt $/ \mathrm{Al}_{2} \mathrm{O}_{3}$, où le cobalt pénètre dans le réseau de l'alumine en se substituant à l'alumine pour former la spinelle $\mathrm{CoAl}_{2} \mathrm{O}_{4}$ [10]. Ceci est important car le cobalt ainsi engagé est inactif en catalyse.

$\mathrm{La}$ réduction de la spinelle $\mathrm{Co}_{3} \mathrm{O}_{4}$ en deux étapes sucessives via l'espèce $\mathrm{CoO}$ a été confirmée par les analyses numériques EXAFS. Une telle réduction peut être également suivie par thermoréduction programmée (TPR) en mesurant la quantité d'hydrogène consommée pendant la réduction. Dans la littérature le profil de thermoréduction programmée (TPR) de $\mathrm{Co}_{3} \mathrm{O}_{4}$ massique est constitué de deux pics bien distincts dont la surface du premier est un tiers plus faible que celle du second [11] conformément aux réactions:

$$
\begin{aligned}
& \mathrm{Co}_{3} \mathrm{O}_{4}+\mathrm{H}_{2}-->3 \mathrm{CoO}+\mathrm{H}_{2} \mathrm{O} \\
& 3 \mathrm{CoO}+3 \mathrm{H}_{2}-\rightarrow 3 \mathrm{Co}^{\circ}+3 \mathrm{H}_{2} \mathrm{O}
\end{aligned}
$$

Cependant, dans le cas de catalyseurs supportés sur silice, le profil TPR ne permet pas d'identifier distinctement les deux étapes de réduction et donc les espèces mises en jeu (CoO et $\mathrm{Co}^{\circ}$ ). La dispersion des particules de cobalt, l'homogénéité ou l'hétérogénéité en taille de ces particules ainsi que la texture du support, modifient le profil TPR [12]. L'analyse EXAFS du catalyseur $25 \% \mathrm{Co} / \mathrm{SiO}_{2}$ met en évidence la présence simultanée à $553 \mathrm{~K}$ des deux structures $\mathrm{CoO}$ et $\mathrm{Co}_{\text {métal }}$. L'étape notée (2) semble être ralentie cinétiquement. Deux explications sont possibles pour expliquer ce phénomène. Pour une texture microporeuse du 
support (surface spécifique du catalyseur: $393 \mathrm{~m}^{2} / \mathrm{g}$ l'eau de réduction formée aura des difficultés à quitter rapidement la surface; ceci ayant pour effet d'inhiber en partie le processus de réduction [13]. L'hétérogénéité des tailles des particules de cobalt, observée dans notre cas par MET, est une autre raison possible de la présence simultané des deux espèces $\mathrm{CoO}$ et $\mathrm{Co}^{\circ}$. Les petites particules d'oxyde de cobalt sont en général plus difficilement réductibles [12] que les grandes.

Les études menées en spectroscopie des photoélectrons (XPS) sur ce type de système catalytique [14], montrent l'évolution en surface du catalyseur au cours de la réduction; les deux étapes discutées plus haut sont également confirmées par cette technique.

Après réduction à $673 \mathrm{~K}$, le catalyseur $25 \% \mathrm{Co} / \mathrm{SiO}_{2}$ est réduit dans sa totalité. Tout comme l'ont remarqué Huffman et al. [15], le nombre de coordination Co-Co $(\mathrm{N}=7,8)$ du catalyseur $\mathrm{Co} / \mathrm{SiO}_{2}$ réduit est plus faible que celui de la feuille de cobalt pris en référence $(\mathrm{N}=12)$. Ceci est attribuable d'une part à la taille des cristallites de cobalt et d'autre part au fait que le catalyseur est supporté.

D'un point de vue catalytique, l'activation du catalyseur sous hydrogène permettant d'accéder à une phase uniquement métallique, sans interaction avec le support de silice (pas de formation de silicate de cobalt), optimise le potentiel du système catalytique en synthèse $\mathrm{CO} / \mathrm{H}_{2}$.

\section{CONCLUSION}

La spectroscopie d'absorption $\mathrm{X}$ est un outil de choix pour connaître, de manière quantitative, l'état de réduction du cobalt dans le bulk. Elle nous a également permis d'accéder à une mesure précise des rayons de la première sphère de coordination du cobalt et du nombre d'atomes contenus dans cette sphère.

Les résultats obtenues en spectroscopie d'absorption $X$ sont complémentaires des études XPS et TPR. Ils confirment l'existence d'une réduction de $\mathrm{Co}_{3} \mathrm{O}_{4} / \mathrm{SiO}_{2}$ en deux étapes dans le bulk:

$$
\mathrm{Co}_{3} \mathrm{O}_{4} \stackrel{\mathrm{H}_{2}}{\rightarrow} \mathrm{CoO} \stackrel{\mathrm{H}_{2}}{\rightarrow} \mathrm{Co}_{\text {métal }}
$$

L'extension de ce travail à un traitement in situ sous flux réactionnel $\left(\mathrm{CO} / \mathrm{H}_{2}\right)$ permettra une investigation sur la structure de la phase active sur notre catalyseur, ce qui est l'un des éléments clé de la compréhension du mécanisme global de la réaction et de l'amélioration des propriétés catalytiques.

\section{REMERCIEMENTS}

Cette étude a été développée dans le cadre du programme COST (N DS/0001/93). Nous remerçions Mme Françoise Villain et Monsieur Dominique Bazin, du Centre du Rayonnement Synchrotron LURE (Orsay) ainsi que Monsieur J. L. Schmitt, L.E.R.C.S.I. (Strasbourg), pour leur aide dans la réalisation des mesures EXAFS.

\section{REFERENCES}

[1] Evans J. , in Catalysis (Bond G. C., Webb G., 1989) vol 8 R. Soc. of Chemistry, Cambridge.

[2] Joyner R.W., in Fundamental Aspects of Heterogeneous Catalysis Studied by Particle Beams (H. H. Brongersma, R. A. van Santen Plenum, New York, 1991) pp 191.

[3] Bartholomew C. H. Catal. Let. , 7 (1990) 303-316.

[4] Somojai G. A. and Thomas J. M., Fischer-Tropsch and methanol synthesis, Topics in Catalysis 2 (1995) 1-311.

[5] Goodwin J. G. Preprints -Symposia American Chemical Society, 36 (1991) 156-159.

[6] Brunauer S., Emmett P. H. and Teller E., J. Amer. Chem. Soc., 60 (1938) 309.

[7] Klug H. P., Alexander L. E., X-Ray Diffraction Procedures for Polycristalline and 
Amorphous Materials, (Wiley-Interscience 1974 New York) 634-642.

[8] Teo B. K., in EXAFS: Basic Principles and Data Analysis (Springer-Verlag, Berlin, 1986).

[9] Lapidus A., Krylova A., Kazanskii V., Borovskov V., Zaitsev A., Rathousky J., Zukal A. and Jancalkova M., Appl. Catal., 73 (1991) 65-82.

[10] Chin R. L. and Hercules D. M., J. Phys. Chem., 86 (1982) 360-367.

[11] Sexton B. A., Hughes A. E. and Turney T. W., J. Catal., 97 (1986) 390-406.

[12] D.G. Castner, P.R. Watson and I. Y. Chan, J. Phys. Chem., 94 (1990) 819-828.

[13] N. W. Hurst, S. J. Gentry, A. Jones and B. D. McNicol, Catal. Rev. -Sci. Eng., 24 (2) (1982) 233-309.

[14] Ernst B., Hilaire L. and Kiennemann A., unpublished results.

[15] Huffman G. P., Shah N., Zhao J., Huggins F. E., Hoost T. E., Halvorsen S. and Goodwin J. G., J. Catal., 151 (1995) 17-25. 